\title{
Research on image sensitive information recognition based on machine learning
}

\author{
LI Kai-yong ${ }^{1}$, MAO Duo-lu ${ }^{2}$ \\ \{WEIYI220@tom.com¹, zhushuang183@163.com²\} \\ ${ }^{1,2}$ Qinghai Nationalities University Physics and Electronic Information Engineering, Xining, 810007 \\ China;
}

\begin{abstract}
Aiming at the problem of low accuracy in traditional image sensitive information recognition methods, a new image sensitive information recognition method based on machine learning was proposed. The pre-processing operations of de-noising and detail sharpening are carried out for the recognition image, and the pre-processing image features are extracted from the three perspectives of color, shape and texture. The image sensitive information is retrieved by combining the extracted image features. Based on the principle of support vector machine (SVM) in machine learning, an image classifier is designed to realize the classification and recognition of image sensitive information. Compared with the two traditional image recognition methods, it is proved that this method has higher precision, shorter recognition time and is more suitable for the recognition of image sensitive information.
\end{abstract}

Key words: machine learning; Image sensitive information; Identification method; Support vector machine;

\section{Introduction}

With the rapid development of Internet technology and mobile communication technology, people's access to information becomes more convenient and diversified. There are a lot of sensitive information mixed in these information, including pornography, violence, contraband, cult and reactionary sensitive content information, which brings a huge challenge to the information security review of the network information regulatory department. Image recognition is widely used in real life, and image recognition technology is gradually improved with the development of science and technology. Images are closely related to human life [1-2]. As the main source of information about the external world, people rely more and more on pictures for communication, which brings convenience to life and highlights the importance of visual technology. People's dependence on network communication makes the amount of image information on the network increase continuously. With the continuous development of network and other related technologies, a large number of images containing sensitive information are widely spread on the network, which has a 
negative impact on social development. Therefore, the research on image sensitive information recognition methods is more and more important [3-4]. The traditional image sensitive information recognition method uses emotion to distinguish, mainly for the text information contained in the image to identify, has certain limitations. The image sensitive information recognition method based on image feature extraction needs to compare the image features several times in the process of recognition.

Machine learning is an interdisciplinary subject involving probability theory, statistics, approximation theory, convex analysis, algorithm complexity and other theories. Machine learning is to simulate the human brain, so that the machine can learn autonomously just like human, so as to acquire new knowledge or skills, and reorganize the existing knowledge structure to continuously improve its performance [5]. Human mainly obtains the surrounding things through vision, presents the images through the eyes, and finally forms relevant concepts in the brain through certain abstraction and reasoning [6]. Machine vision in machine learning is to use machines to simulate the human brain, obtain pictures through equipment, and then after certain processing and feature extraction, finally identify relevant information. The core of machine learning is the training of classifier [7]. Through continuous training and adjustment, a good classifier model is finally obtained to realize accurate recognition of things. The good learnability of machine learning means that this method can be widely applied in the field of image recognition [8]. Based on the above analysis, this paper proposes an image sensitive information recognition method based on machine learning.

\section{Research on image sensitive information recognition method based on machine learning}

\subsection{Image preprocessing}

For the image to be recognized, image denoising and image detail sharpening are required. Since certain noise points will be included in the image, the existence of noise points will affect the accuracy of image feature extraction and subsequent recognition, so the noise needs to be filtered out before identifying sensitive information of the image [9-10].

In this paper, the image is denoised by means of mean filter and median filter. Let

$r(x, y)$ be the noisy image to be processed, and the image processed by mean filtering is $q(x, y)$, which can be expressed by the following formula.

$$
q(x, y)=\frac{1}{K} \sum r(x, y),(x, y) \in \Omega
$$

In formula (1), $\Omega$ is the set of coordinates of all pixel points in the window 
neighborhood calculated by the mean filter template, and $K$ is the total number of all pixel points in the window neighborhood. Mean filtering is suitable for denoising images with larger Windows, that is, images with larger sizes [11]. However, mean filtering can erase certain image details. Therefore, median filtering technology is used to filter images.

Set the center point of the image as $\left(x_{0}, y_{0}\right)$, arrange all pixel points in the neighborhood centered on point $\left(x_{0}, y_{0}\right)$ in descending order according to the gray value of the pixel, and take the middle value after sorting as the current pixel point, that is, the center point of the neighborhood. The $3 \times 3$ template was used to replace the original gray value of the central pixel with the median value, and the median value was filtered to obtain the image $f(x, y)$ after two filters, and the denoising of the image was completed [12].

After denoising the image, the boundary contour of the image will be blurred, so the image needs to be sharpened. For denoising image $f(x, y)$, the gradient at $(x, y)$ of a certain pixel in the image is represented as vector $G$.

$$
G=[f(x, y)]=\left[\frac{\partial f}{\partial x}, \frac{\partial f}{\partial y}\right]
$$

The direction of vector $G$ gradient is the maximum change rate of $f(x, y)$, and the difference processing method is used to calculate the gradient of image $f(x, y)$. After calculating the gradient, the following formula is used to further correct the gradient.

$$
g(x, y)=\left\{\begin{array}{l}
G[f(x, y)], \quad G(f) \geq T \\
f(x, y), \text { else }
\end{array}\right.
$$

In formula (4), $T$ is the gradient threshold, and $T$ is a non-negative value. An appropriate threshold is selected to highlight the edge contour of the object to be examined in the image [13]. After image preprocessing, image features are extracted.

\subsection{Image feature extraction}

Image feature is the key of image recognition. Image feature can be divided into global feature and local feature. In this paper, image features are extracted from color, shape and texture. Color histogram is used to extract the color features of the image. Color histogram is 
to calculate the frequency of each gray scale in the color [14-15]. The gray scale of color histogram represents the color brightness of the image. The horizontal coordinate represents the gray scale of the image $L$, and the vertical coordinate represents the probability of gray scale appearing in the image $p(l)$. The calculation formula of color histogram is as follows:

$$
p(l)=\frac{n_{l}}{N}, l=0,1,2 \cdots, L-1
$$

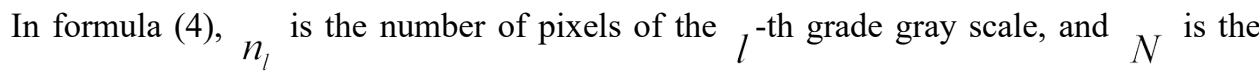
sum of pixels in the image. After the color features of the image are extracted, shape features in the image are further developed. In this process, the edges of things in the image need to be detected first [16].

The Sobel operator is adopted to image edge detection, Sobel operator is a discrete convolution template, according to the brightness of the image channel matrix for convolution operation, detecting the edge of the object in the image to find the boundary of the pixel values mutations, Sobel operator is through the horizontal, vertical and two direction of image edge detection, so you need to $x, y$ convolution template in two directions, as shown below:

$$
\begin{gathered}
G_{x}=\left[\begin{array}{ccc}
-1 & 0 & 1 \\
-2 & 0 & 2 \\
-1 & 0 & 1
\end{array}\right] \\
G_{y}=\left[\begin{array}{ccc}
1 & 2 & 1 \\
0 & 0 & 0 \\
-1 & -2 & -1
\end{array}\right]
\end{gathered}
$$

According to the above template, the operation process of convolution operation on the image is as follows:

$$
\left\{\begin{array}{l}
I_{x}=G_{x} \times A \\
I_{y}=G_{y} \times A
\end{array}\right.
$$

In formula (7), $I_{x}$ and $I_{y}$ are the gray values of edge detection in the horizontal and vertical directions of the image respectively, and 6 are the image brightness channel matrix [17-18]. The calculation formula of each gray value of the final edge detection matrix is as follows: 


$$
G=\sqrt{G_{x}^{2}+G_{y}^{2}}
$$

After detecting the edges in the image, shape features in the image are extracted according to the edge feature map. Rotate from $0^{\circ}$ to $360^{\circ}$ with the image center as the origin, count the number of image pixel points whose gray value is not 0 on each Angle, draw the histogram with the Angle as the abscissa and the number as the ordinate, and normalize the histogram to make the distribution of the resulting histogram more uniform [19]. After the shape feature of the image is obtained from the histogram, the image texture feature is extracted.

Texture is an important visual feature of the image, because the change of gray level of the image shows certain rules. Texture shows the spatial information of image internal structure and pixel distribution [20-21]. In this paper, gray co-occurrence matrix algorithm is used to extract image texture features.

For a complete image, it can be seen that there are $n$ points in the horizontal direction, represented by $1,2,3, \cdots, n$ and ${ }_{N}$ respectively. There are $m$ points in the vertical

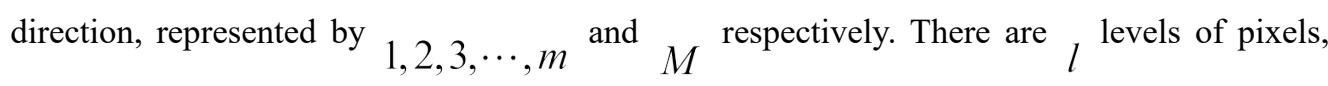
$1,2,3, \cdots, l$ respectively. $L^{\prime}$ is used to represent the level. Therefore, the image can be regarded as a gray matrix of $M \times N->L^{\prime}$.

After the image is converted into gray image, the gray image is quantized according to the size of the image. If the gray level co-occurrence matrix of the quantized gray level image is $P$, take the specific direction $w$, and the value of direction $w$ is 0 degree, 45 degree, 90 degree and 135 degree [22]. The texture parameters are then calculated.

Image texture energy represents the thickness of texture. The larger the energy value is, the stronger the texture vein is. The smaller the energy value is, the more detailed the texture vein is. The calculation formula of image texture energy is as follows:

$$
F_{1}=\sum_{i, j} p(i, j)^{2}
$$

In formula (9), $F_{1}$ is the image texture energy, $p(i, j)$ is the probability that the gray level of the image appears in a unit distance in a specific direction from level $i$ to level $j$ [23]. At the same time, the moment of inertia can also represent the thickness of texture. The formula of moment of inertia $\mathrm{F}_{2}$ is as follows: 


$$
F_{2}=\sum_{i, j}(i-j)^{2} p(i, j)^{2}
$$

It can be seen from the above formula that the smaller the value of $F_{2}$, the stronger the texture vein of the image; the larger the value of $F_{2}$, the more detailed the texture vein of the image. Entropy is used to express the complexity of image texture, and its calculation formula is as follows:

$$
F_{3}=\sum_{i, j} p(i, j) \log _{2} p(i, j)
$$

It can be seen from the above formula that the smaller the entropy value of $F_{3}$ is, the simpler the texture is, the larger the value is, and the more complex the texture is. In order to recognize the sensitive information in the image, the similarity degree of the row and column directions of the texture in the image is calculated according to the following formula.

$$
F_{4}=\frac{\sum_{i, j} i \times j \times \log _{2} p(i, j)-\mu_{x} \times \mu_{y}}{\sigma_{x} \times \sigma_{y}}
$$

In the above formula, $\mu_{x}$ and $\mu_{y}$ are mean values, and $\sigma_{x}$ and $\sigma_{y}$ are variance. To sum up, the texture features of the image can be obtained by calculating the mean and variance of $F_{1}, F_{2}, F_{3}$ and $F_{4}$. After extracting the features of the image, the sensitive information contained in the image features is retrieved.

\subsection{Image sensitive information retrieval}

Image sensitive information can be divided into illegal bad information, involving personal privacy, images containing exposure behavior and text embedded in the image. Therefore, according to the image features extracted above, the image to be recognized is roughly divided into two categories, including text and not including text, and sensitive information retrieval is carried out respectively.

For the image sensitive information retrieval without text, the fusion convolution neural network and image color feature algorithm are used first, and the specific process of the algorithm is shown in the Figure 1. 


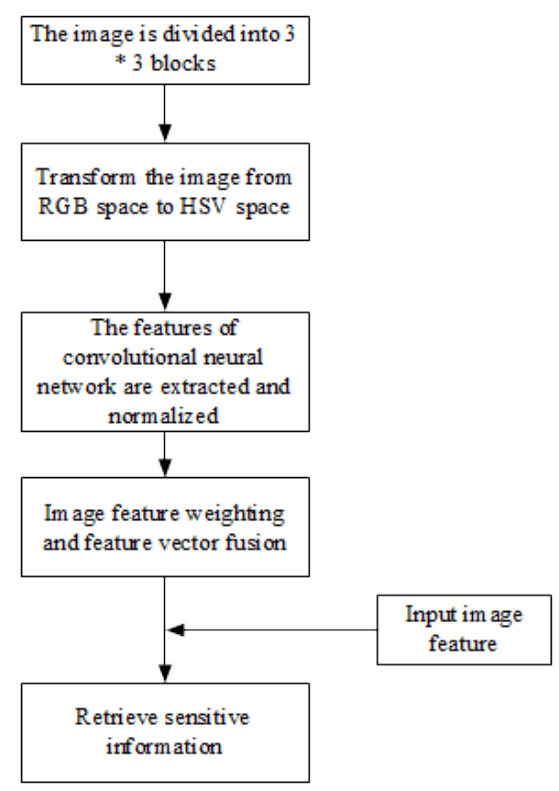

Fig. 1 Flow chart of fusion convolution neural network and image color feature algorithm

Based on the color features extracted in the above section, in order to further characterize the sensitive information in the image, this paper uses the first-order moment, second-order moment and third-order moment of color to further characterize the color features. After the image is transformed from RGB space to HSV space, the first-order moment, second-order moment and third-order moment can be used as elements in a row of three column vectors to represent the color moment vector of a certain channel of the image.

The first-order moment value is the mean value of all elements of a channel matrix, the second-order central moment is the pixel variance of the image channel, and the third-order moment is the cube root of the ratio of the difference cubic matrix value of the channel to the total number of image pixels. Three channel vectors in HSV space are sequentially spliced, and the vector of image color moment is finally described as a vector with 1 row and 9 columns. Thus, the expression of normalized color feature vector of HSV spatial model is obtained as follows:

$$
R_{i}=\frac{V_{i}}{\sqrt{\sum_{i=1}^{N^{\prime \prime}} V_{i}^{2}}}
$$

Where, $i_{i}$ represents the number of elements in the color feature vector, $R_{i}$ represents the 


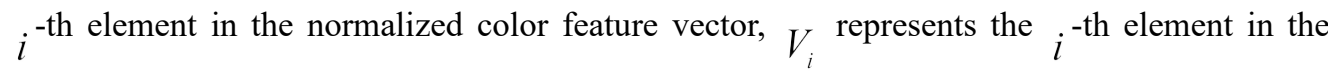
color feature vector before normalization, and $N^{\prime \prime}$ represents the total number of elements in the vector. After the retrieval algorithm retrieves the sensitive information in the image, the image is input into the image sensitive information classifier, waiting for further processing.

The retrieval of image sensitive information containing text is mainly carried out by the texture width and color feature of a certain part of the image. In general, the text area in the image has relatively stable texture width and relatively uniform color features. According to the image features extracted above, the image containing text is screened. Sensitive information character detection based on context content is carried out for the screened images.

In order to save the operation cost of this method, the convolutional neural network is used to detect the sensitive characters in the image. The sample set of sensitive characters was established, which was used to train CNN and determine the parameters of the convolutional neural network. Assuming the training set is $\left\{\left(x^{(1)}, y^{(1)}\right), \cdots,\left(x^{(m)}, y^{(m)}\right)\right\}$, the neural network parameters are trained by the gradient descent method. The loss function of the whole sample set is defined as $J(W, b)$, and the local minimum value is obtained by using the gradient iteration method, i.e.

$$
\left\{\begin{array}{l}
W_{j i}^{(l)}=W_{j i}^{(l)}-\alpha \frac{\partial}{\partial W_{j i}^{(l)}} J(W, b) \\
b_{i}^{(l)}=b_{i}^{(l)}-\alpha \frac{\partial}{\partial b_{i}^{(l)}} J(W, b)
\end{array}\right.
$$

Where, $\alpha$ is the learning rate of the convolutional neural network. After determining the parameters of the convolutional neural network, the character detector is used for sliding detection, and the region containing text is extracted from the image. The similarity between the detected characters in this region and the elements in the sample set of sensitive information is calculated. If the similarity is greater than 0.5 , the character sensitive information in the image is determined to be retrieved. If the similarity is less than 0.5 , the sensitive information of character class is not detected. After the sensitive information in the image is detected, it is further processed in the classifier to complete the image sensitive information recognition.

2.4 Image sensitive information recognition based on machine learning 
In this paper, the SVM principle in machine learning is adopted to design an image classifier and realize the recognition of image sensitive information. The kernel function and penalty coefficient are the core parts of SVM. The kernel function is to map the linear indivisible vectors of low dimension to the linear separable vector space of high dimension. Penalty factors can be adjusted accordingly when the samples are misclassified to reduce the case of misclassification, so the generalization ability of the classifier should be guaranteed.

SVM first performs classification calculation in low-dimensional space, then maps lowdimensional space to high-dimensional feature space, constructs optimal hyperplane in highdimensional feature space, and separates data. The conversion process from $2 \mathrm{~d}$ dataset to $3 \mathrm{~d}$ space is shown in Figure 2.
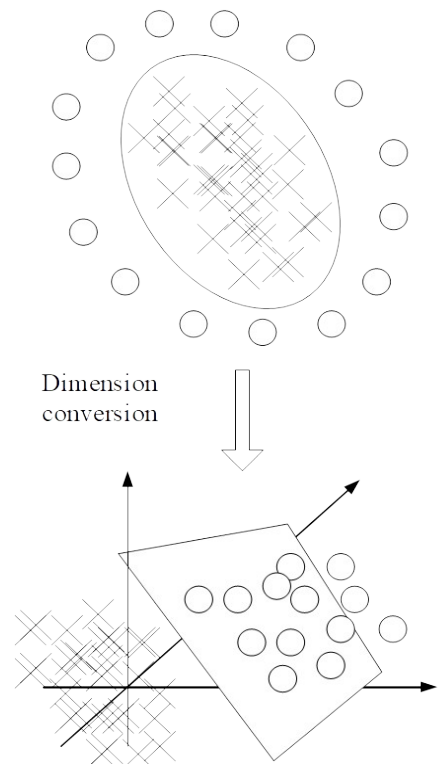

Fig. 2 Spatial dimension transformation diagram

The final expression of the SVM can be converted into the following form.

$$
f(x)=\left(\sum_{i=1}^{n} \alpha_{i} y_{i} x_{i}\right)^{T} x+b
$$

In the above equation, $x_{i}$ is the vector in the dataset, $\alpha_{i}$ is the Lagrange multiplier corresponding to dataset $x_{i}$, and $y_{i}$ is the decision character of $x_{i}$. In the process of data set classification, the following classification function is constructed. 


$$
f(x)=\sum_{i=1}^{N} w_{i} \phi_{i}(x)+b
$$

Function $\phi(x)$ converts a dataset from two dimensions to three dimensions. The sensitive information in the image contains multiple categories, so it is necessary to design multiple classifiers for classification. According to the idea of binary tree, the multi-classifier as shown in the figure below is constructed. After $n-1$ classification divisions, the required $n$ categories can be identified.

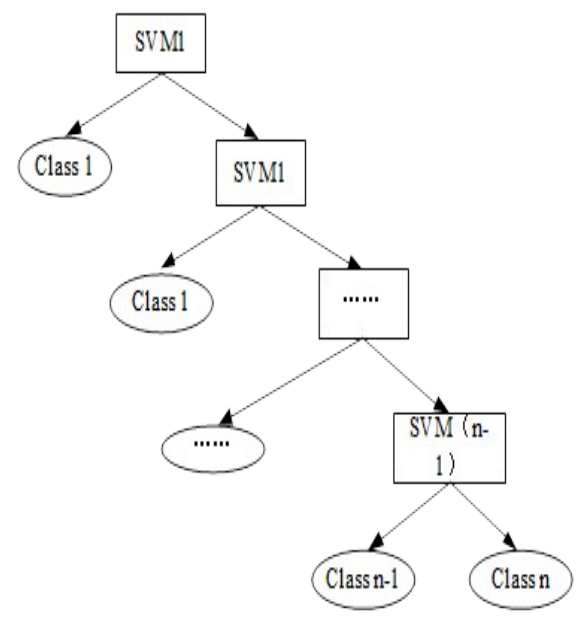

Fig. 3 Binary tree multi-classifier structure

The image sensitive information feature set was used to train the multi-classifier, and the classification parameters of SVM were obtained after the training. Input the image to be recognized, and after the above processing, calculate the similarity between the image and the feature vector of the sample set in the SVM. If not, the classification is stopped to realize the recognition of the sensitive information of the image. So far, the research on the image sensitive information recognition method based on machine learning has been completed.

\section{Experiment}

In order to verify the effectiveness of the image sensitive information recognition method based on machine learning studied in this paper, comparative experiments will be conducted to evaluate the practical application effect of the research method in this paper by analyzing the experimental results.

\subsection{Experimental contents}


In the form of comparative experiment, the comparison group of the experiment is the image recognition method based on principal component analysis and the image recognition method based on template matching, and the test group is the image sensitive information recognition method based on machine learning studied in this paper. The comparison index of the experiment is the precision and recognition time of three different recognition methods, which are used to evaluate the advantages and disadvantages of the three recognition methods. In order to ensure the scientific validity of the experimental results, unique experimental variables were controlled during the experiment to complete the experimental verification.

\subsection{Experimental preparation}

The experiment was completed on three computers with the same configuration. The experiment was divided into three groups according to the data type for experimental verification. The specific parameters of experimental data are shown in the following table.

Table 1 Experimental data parameters

\begin{tabular}{cccc}
\hline Group & The data type & $\begin{array}{c}\text { Total } \\
\text { data }\end{array}$ & Quantity of sensitive information \\
\hline A11 & The image contains clear text & 15680 & 5372 \\
A12 & & 46873 & 21796 \\
A13 & & 3600 & 1276 \\
A21 & Images do not contain text & 22134 & 5341 \\
A22 & & 4236 & 983 \\
A23 & & 1367 & 421 \\
A31 & Image information blur & 3546 & 437 \\
A32 & & 6078 & 1592 \\
A33 & & 10854 & 4375 \\
\hline
\end{tabular}

Three identification methods were used to identify image sensitive information from different experimental data, and the accuracy of the three methods was recorded. The experimental results are as follows.

\subsection{Experimental results}

The experimental results are shown in the following table. By analyzing the data in the table, relevant conclusions of this experiment are drawn.

Table 2 Accuracy rate $(\%)$ of sensitive information by different identification methods

\begin{tabular}{lllll}
\hline $\begin{array}{l}\text { Experimental } \\
\text { data number }\end{array}$ & $\begin{array}{l}\text { Method based on principal } \\
\text { component analysis }\end{array}$ & $\begin{array}{l}\text { Template matching } \\
\text { based approach }\end{array}$ & $\begin{array}{l}\text { This } \\
\text { method }\end{array}$ & paper's \\
\hline A11 & 54.70 & 68.10 & 90.30 & \\
A12 & 62.10 & 62.30 & 82.40 & \\
A13 & 67.60 & 66.50 & 83.80 & \\
A21 & 54.90 & 57.50 & 84.20 & \\
A22 & 62.70 & 65.20 & 86.50 & \\
A23 & 63.50 & 56.30 & 85.60 \\
A31 & 61.60 & 62.40 & 82.40
\end{tabular}


\begin{tabular}{rrrr} 
A32 & 62.50 & 63.70 & 83.20 \\
A33 & 63.90 & 71.40 & 94.10 \\
\hline
\end{tabular}

It can be seen from the above table that the accuracy of the research method in this paper is higher when different experimental data are identified as image sensitive information. In the comparison group, the accuracy of the image recognition method based on principal component analysis is lower than that based on template matching. During the calculation, the average accuracy of the three identification methods was $61.5 \%$ for the identification method based on principal component analysis, $63.7 \%$ for the identification method based on template matching, and $85.83 \%$ for the identification method in this paper.

On the basis of the above experiments, compare the recognition time of different methods, and the results are shown in Figure 4.

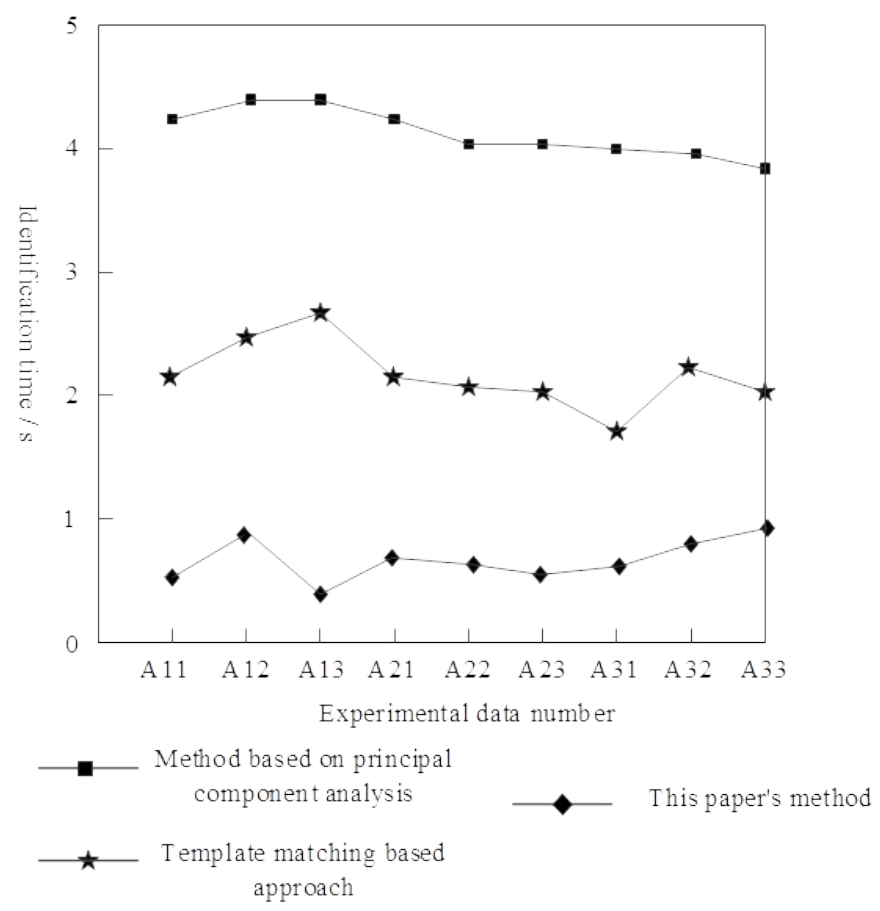

Fig. 4 Comparison of recognition time of different methods

Analysis of the above figure shows that the recognition time of the recognition method based on principal component analysis from $4.0 \mathrm{~s}$ to $4.5 \mathrm{~s}$, and the recognition time of the recognition method based on template matching changes from $1.9 \mathrm{~s}$ to $2.7 \mathrm{~s}$. The recognition time of the method in this paper is always less than $1.0 \mathrm{~s}$, with short recognition time and higher efficiency. 
In conclusion, this method can realize the fast and accurate recognition of image sensitive information.

\section{Conclusions}

In order to solve the problem of low accuracy in traditional image sensitive information recognition methods, a new image sensitive information recognition method based on machine learning is proposed. The pre-processing of denoising and detail sharpening is carried out for the recognition image, and the image sensitive information is retrieved by combining the extracted image features. Based on the principle of SVM in machine learning, an image classifier is designed to realize the classification and recognition of image sensitive information. The simulation results show that this method has higher accuracy, better performance, better practical application and reliability, which provides a new idea for the further development of image sensitive information recognition technology. In the future, with the strengthening of the national government's supervision on the network information security, it is necessary to study the issue that many illegal institutions and individuals use image to release sensitive information, so as to further curb the image transmission with sensitive information, so as to purify the network environment, reduce the transmission of sensitive information on the physical and mental health of young people and social harmony adverse effects of stability.

\section{Fund projects}

science and technology plan of Qinghai province(Key Research\&Development and conversion plans 2019-GX-170)

project name:Development of resource library of duixiu art Image digital protection in Huangzhong County, Qinghai Province

\section{References}

[1] Zuo Zhenyu. Medical image segmentation, registration, fusion, and denoising based on machine learning. Electronic Design Engineering.27(17).135-139 (2019)

[2] Chen Qi ,Zhang Yuehua, Wang Hong . Research on Image Denoising Based on Machine Learning. Modern Informationn Technology. 3(14).71-73 (2019)

[3] Li Zhiyi , Xu Hongkai , Duan Bin. Research on Image Emotion Feature Extraction Based on Deep Learning CNN Model. Library and Information Service.63(11).96-107 (2019)

[4] Li Yixin ,Zou Yajun ,Ma Jinwen . Document Region Image Classification via Feature Extraction and Machine Learning Algorithms. Journal of Signal Processing.35(05).747-757 (2019)

[5] Bao Ruiqing . Research on Intellectual Analysis and Application of Landscape Architecture Based on Machine Learning . Landscape Architecture 26(05).29-34 (2019) 
[6] Liu Yunpeng, Pei Shaotong ,Wu Jianhua, et al. Deep Learning Based Target Detection Method for Abnormal Hot Spots Infrared Images of Transmission and Transformation Equipment . Southern Power System Technology. 13(02).27-33 (2019)

[7] Huang Lye, Wu Lushen, Chen Huawei. Image Blur Types and Parameters Estimation Using DCNN Fusion with the LSTM . Journal of Basic Science and Engineering.26(05).1092-1100 (2018)

[8] Ann Qiangqiang, Zhang Feng, Li Zhaoxing ,et al. Research on image segmentation based on machine learning. Automation \& Instrumentation. 28(06).29-31 (2018)

[9] Tian Zhaohui , Jin Xin, Zhao Geng, et al. A Method for breaking Image CAPTCHA Based on Deep Learning. Computer Simulation.35(03).216-220 (2018)

[10] Zhang Ye , Li Mingchao ,Han Shuai . Automatic identification and classification in lithology based on deep learning in rock images. Acta Petrologica Sinica.34(02).333-342 (2018)

[11] Qiong Li, Tingting Zhao, Lingchao Zhang, et al. Ferrography Wear Particles Image Recognition Based on Extreme Learning Machine. Journal of Electrical and Computer Engineering. 2017(2).1-6 (2017)

[12] Jin Shoufeng, Fan Di, Malekian Reza,et al. An image recognition method for gear fault diagnosis in the manufacturing line of short filament fibres. Insight: Non-Destructive Testing and Condition Monitoring. 60(5).270-275 (2018)

[13] Priyadarshini Panda, Abhronil Sengupta, Kaushik Roy. Energy-Efficient and Improved Image Recognition with Conditional Deep Learning. ACM Journal on Emerging Technologies in Computing Systems.13(3).1-21 (2017)

[14] Hong-wei Huang, Qing-tong Li, Dong-ming Zhang. Deep learning based image recognition for crack and leakage defects of metro shield tunnel. Tunnelling \& Underground Space Technology.77(4).166-176 (2018)

[15] A.V. Savchenko. Maximum-likelihood dissimilarities in image recognition with deep neural networks. Computer Optics.41(3).422-430 (2017)

[16] A.V. Savchenko. Maximum-likelihood approximate nearest neighbor method in real-time image recognition. Pattern Recognition.61(3).459-469 (2017)

[17] Hu, T H, Wan, L, Liu, T A,et al. Advantages and Application Prospects of Deep Learning in Image Recognition and Bone Age Assessment. Journal of Forensic Medicine.14(5).629-634 (2017)

[18] Andrey V. Savchenko. Probabilistic Neural Network with Complex Exponential Activation Functions in Image Recognition using Deep Learning Framework. IEEE Transactions on Neural Networks and Learning Systems.PP(99).1-12 (2017)

[19] X.-L. Tang, Y.-M. Du, Y.-W. Liu,et al. Image Recognition With Conditional Deep Convolutional Generative Adversarial Networks. Zidonghua Xuebao/acta Automatica Sinica.44(5).855-864 (2018) 
[20] Zheng Zhang, Yong Xu, Ling Shao, et al. Discriminative Block-Diagonal Representation Learning for Image Recognition. IEEE Transactions on Neural Networks \& Learning Systems.PP(99).1-15 (2017)

[21] Shan Wang, YueXing Wang, HuiLing Shan,et al. Multimodal computer image recognition based on depth neural network. Cluster Computing.45(5).1-7 (2018)

[22] Qianqian Wang, Quanxue Gao, Xinbo Gao,et al. p-norm based PCA for Image Recognition. IEEE Transactions on Image Processing.PP(99).1-1 (2017)

[23] Xiao-Bo Zhang, Xiao-Guang Ge, Yan Jin, et al. Application of image recognition technology in census of national traditional Chinese medicine resources. Chinese materia medica.42(22).4266-4270 (2017) 